\title{
Cost-Minimization Analysis of Pembrolizumab Monotherapy Versus Nivolumab in Combination with Ipilimumab as First-Line Treatment for Metastatic PD-L1-Positive Non-small Cell Lung Cancer: A US Payer Perspective
}

\author{
Nan Qiao $^{1}$ (D) Ralph Insinga ${ }^{1} \cdot$ Thomas Burke $^{2} \cdot$ Gilberto Lopes $^{3}$
}

Accepted: 9 July 2021 / Published online: 22 July 2021

(c) The Author(s) 2021, corrected publication 2021

\begin{abstract}
Background Pembrolizumab monotherapy and nivolumab in combination with ipilimumab are US FDA-approved first-line (1L) regimens for patients with metastatic non-small cell lung cancer (NSCLC) without epidermal growth factor receptor or anaplastic lymphoma kinase genomic aberrations and with a programmed death ligand 1 (PD-L1) tumor proportion score (TPS) of $\geq 1 \%$. A published matching-adjusted indirect comparison found the two regimens yield comparable overall and progression-free survival outcomes.

Objective The aim of this study was to compare direct medical costs of pembrolizumab and nivolumab plus ipilimumab for PD-L1-positive metastatic NSCLC treatment within the first 3 years following treatment initiation from a US payer perspective.

Methods A cost-minimization model was built to estimate and compare treatment, disease management, and adverse event costs based on KEYNOTE-024 and -042, and CheckMate 227 Part 1a trial survival and adverse event data.

Results $1 \mathrm{~L}$ pembrolizumab generates $\$ 54,343, \$ 75,744$, and $\$ 76,259$ per patient cost savings compared with $1 \mathrm{~L}$ nivolumab plus ipilimumab for patients with NSCLC with PD-L1 TPS $\geq 1 \%$ within 1, 2, and 3 years of treatment initiation, respectively. Conclusion Pembrolizumab is cost saving as $1 \mathrm{~L}$ treatment for PD-L1-positive metastatic NSCLC in comparison with nivolumab plus ipilimumab, at least for the short term.
\end{abstract}

Nan Qiao

nan.qiao@merck.com

Ralph Insinga

ralph_insinga@merck.com

Thomas Burke

thomas_burke2@merck.com

\section{Key Points for Decision Makers}

Gilberto Lopes

glopes@med.miami.edu

1 Biostatistics and Research Decision Sciences, Merck \& Co., Inc., 2000 Galloping Hill Road, Kenilworth, NJ 07033, USA

2 Center for Observational and Real-World Evidence, Merck \& Co., Inc., 2000 Galloping Hill Road, Kenilworth, NJ 07033, USA

3 Sylvester Comprehensive Cancer Center at the University of Miami, 1120 NW 14th St, Suite 650J, Miami, FL 33136, USA

Pembrolizumab is cost saving compared with nivolumab in combination with ipilimumab for first-line programmed death ligand 1-positive metastatic non-small cell lung cancer.

Most of the cost savings are attributable to drug acquisition costs.

\section{Introduction}

Programmed cell death protein 1 (PD-1) inhibitors have transformed the landscape of metastatic non-small cell lung cancer (NSCLC) treatment. Pembrolizumab monotherapy was approved by the US FDA for the first-line (1L) treatment of metastatic NSCLC or stage III NSCLC not eligible 
for surgical resection or chemoradiation, without epidermal growth factor receptor $(E G F R)$ or anaplastic lymphoma kinase $(A L K)$ genomic tumor aberrations, and with a programmed death ligand 1 (PD-L1) tumor proportion score (TPS) of $\geq 1 \%$ based on the KEYNOTE-024 study (TPS $\geq 50 \%$ ) in 2016 and KEYNOTE-042 study (TPS $\geq 1 \%$ ) in 2019 [1]. Nivolumab in combination with the cytotoxic T-lymphocyte-associated protein 4 (CTLA-4) inhibitor ipilimumab was approved by the FDA in 2020 as a $1 \mathrm{~L}$ treatment of metastatic NSCLC with TPS $\geq 1 \%$ and with no EGFR or $A L K$ genomic aberrations based on the results from the CheckMate 227 Part 1a study [2]. Pembrolizumab in combination with platinum-based chemotherapy and nivolumab plus ipilimumab and two cycles of platinum-based chemotherapy have also been approved as a $1 \mathrm{~L}$ treatment for metastatic NSCLC irrespective of PD-L1 expression level [1, 2].

Pembrolizumab monotherapy is recommended by the National Comprehensive Cancer Network (NCCN) as a preferred $1 \mathrm{~L}$ regimen for treating patients with metastatic NSCLC with PD-L1 TPS $\geq 50 \%$ (category 1: indicating $\geq$ $85 \%$ of the NCCN NSCLC panel vote) and is considered useful in certain circumstances for metastatic NSCLC with TPS $1-49 \%$ (category $2 \mathrm{~B}$ : indicating $50-85 \%$ of the vote). Nivolumab in combination with ipilimumab is considered useful in certain circumstances for metastatic NSCLC with either PD-L1 TPS $\geq 50 \%$ or $1-49 \%$ (category 1) [3].

No randomized controlled trials (RCTs) directly compare the efficacy of pembrolizumab monotherapy versus nivolumab plus ipilimumab as $1 \mathrm{~L}$ treatments for PDL1-positive metastatic NSCLC. Halmos et al. [10] conducted an anchored matching-adjusted indirect comparisons (MAIC) study to compare the two regimens based on the CheckMate 227 Part 1a results published by Hellmann et al. [4] and KEYNOTE-024 and -042 clinical trial data $[5,6]$. In this analysis, individual patient data (IPD) from the KEYNOTE-024 and -042 clinical trials were reweighted using a logistic model suggested by Signorovitch et al. [7] to match patients' baseline characteristics such as age, sex, geographic region, Eastern Cooperative Oncology Group (ECOG) performance status score, smoking status, tumor histologic type, metastatic sites, and PD-L1 status reported by Hellmann et al. [4]. The outcomes of interest, progression-free survival (PFS) and overall survival (OS), were analyzed using two approaches. The first approach used treatment effects, log (hazard ratio $[\mathrm{HR}]$ ), and standard errors estimated from a weighted Cox model on KEYNOTE-024 and 042 trial data, as well as the aggregated results reported by Hellmann et al. [4] to perform an indirect treatment comparison (ITC) based on the study by Bucher et al. [8]. The second approach reconstructed IPD from CheckMate 227 Part 1a by employing the algorithm developed by Guyot et al. [9] and then used a Cox proportional hazards model on the weighted
IPD from the KEYNOTE-024 and -042 trials and reconstructed IPD from CheckMate 227 Part 1a to estimate the treatment effect of pembrolizumab versus nivolumab plus ipilimumab. Data from KEYNOTE-024 and -042 were truncated at the maximum follow-up time in CheckMate 227 Part 1a to adjust for differences in follow-up. Results showed that the two regimens had comparable efficacy: PFS HR 1.16 (95\% confidence interval [CI] 0.93-1.45) and OS HR 1.07 (95\% CI 0.82-1.39) using the first approach; and PFS HR 1.12 (95\% CI 0.90-1.39) and OS HR 1.05 (95\% CI 0.84-1.31) using the second approach [10]. Adverse event (AE) comparisons were not included in the study by Halmos et al. due to potential biases in AE collection, reporting, and follow-up between trials [10]. Another identified ITC comparing the two regimens was a network meta-analysis (NMA) conducted by Zhou et al. [11]. Published summary results of KEYNOTE-042 and CheckMate 227 Part 1a were used as inputs in the ITC for the comparison between pembrolizumab and nivolumab plus ipilimumab. PFS and OS HRs were estimated using inversevariance weighting, and treatment-related AEs (TRAEs) were compared using the Mantel-Haenszel method. The study found no significant differences in OS, with an HR of 0.98 (95\% CI 0.77-1.24) for nivolumab plus ipilimumab versus pembrolizumab, consistent with the findings by Halmos et al. [10, 11]. The PFS HR of nivolumab plus ipilimumab versus pembrolizumab was estimated to be 0.77 (95\% CI 0.62-0.95) by Zhou et al. indicating a larger and statistically significant difference in treatment effects on PFS between the two regimens compared with what was detected by Halmos et al. [10, 11]. We speculate the different finding in PFS might be due to the following reasons: the MAIC conducted by Halmos et al. reduced cross-trial differences by adjusting the IPD of KEYNOTE-024 and -042 to match the baseline characteristics of patients in CheckMate 227 Part 1a, making its HR estimates more robust than the HRs estimated by Zhou et al. [10, 11]. In addition, pembrolizumab's treatment effect on PFS was stronger in KEYNOTE-024 (which was not included by Zhou et al.) than in the KEYNOTE-042 TPS $\geq 50 \%$ patient population, which might also partially contribute to the statistically significant PFS benefit of nivolumab plus ipilimumab reported by Zhou et al. $[5,6,11]$. The TRAE comparison by Zhou et al. found that nivolumab plus ipilimumab had a higher incidence of any grade or grade 3 or higher TRAEs (relative risk [RR] 1.28, 95\% CI 1.17-1.40 and RR 2.18, 95\% CI 1.70-2.80, respectively). Among TRAEs of any grade, the incidences of diarrhea, pruritus, fatigue, decreased appetite, asthenia, nausea, and vomiting were significantly higher for nivolumab plus ipilimumab than for pembrolizumab. Among grade 3 or higher TRAEs, only the incidence of fatigue was significantly higher for nivolumab plus ipilimumab versus pembrolizumab [11]. 
A recent literature review identified five cost-effectiveness analyses (CEAs) comparing pembrolizumab monotherapy with the platinum doublet chemotherapy comparators in the KEYNOTE-024 study (i.e. carboplatin/cisplatin plus pemetrexed, carboplatin/cisplatin plus gemcitabine, or carboplatin plus paclitaxel) and the KEYNOTE-042 study (i.e. carboplatin plus pemetrexed or carboplatin plus paclitaxel) from a US payer perspective [12]. Despite variable results due to methodological differences, these CEAs concluded that pembrolizumab was cost effective compared with platinum doublet chemotherapy when treating PD-L1 TPS $\geq 1 \%$ and $\geq 50 \%$ NSCLC patients at a willingnessto-pay (WTP) threshold of $\$ 150,000$ per quality-adjusted life-year (QALY), except the study by She et al. who found an incremental cost-effectiveness ratio (ICER) of $\$ 179,530$ per QALY for PD-L1 TPS $\geq 1 \%$ NSCLC patients [12-16]. Four published CEAs have compared nivolumab plus ipilimumab versus the CheckMate 227 Part 1a trial chemotherapy comparator (carboplatin/cisplatin plus pemetrexed for non-squamous histology, and carboplatin/cisplatin plus gemcitabine for squamous histology) from a US payer perspective. Berling et al. reported an ICER of $\$ 104,385$ per QALY for nivolumab plus ipilimumab versus trial chemotherapy [17], while Courtney et al. estimated an ICER of $\$ 413,400 /$ QALY and suggested a $67 \%$ reduction in treatment costs for the nivolumab plus ipilimumab combination treatment to achieve cost effectiveness at a WTP threshold of $\$ 100,000$ per QALY [18]. Hu et al. concluded that nivolumab plus ipilimumab is cost effective for treating patients with advanced NSCLC with PD-L1 TPS $\geq 1 \%$ and $\geq 50 \%$ over a 20 -year time horizon (ICER $=\$ 107,404$ and $\$ 133,732$ per QALY, respectively) [19]. Finally, Li et al. found that nivolumab plus ipilimumab is not cost effective for treating advanced NSCLC with PD-L1 TPS $\geq 1 \%$ or $\geq 50 \%$ over a 10 -year time horizon (ICER $=\$ 196,507$ and $\$ 212,111$ per QALY, respectively) [20]. In summary, despite varying methodology across CEAs, the majority of analyses concluded that both pembrolizumab monotherapy and the combination of nivolumab and ipilimumab are cost effective when compared with the respective chemotherapy regimens used as control arms in KEYNOTE-024, KEYNOTE-042, and CheckMate 227 Part 1a. No CEAs comparing pembrolizumab and nivolumab plus ipilimumab have been published to date.

Given the assumed comparable efficacy of the two regimens in patients with TPS $\geq 1 \%$ metastatic NSCLC, we conducted a modeling-based cost-minimization analysis (CMA) to compare the direct medical costs of pembrolizumab monotherapy versus nivolumab plus ipilimumab for 1L treatment of patients with PD-L1-positive metastatic NSCLC in the US.

\section{Methods}

\subsection{Target Population}

The study population was patients with metastatic NSCLC who had not previously received systemic therapy and whose tumors express PD-L1 on $\geq 1 \%$ of tumor cells (TPS $\geq 1 \%$ ).

\subsection{Comparators}

The model compared pembrolizumab monotherapy versus the combination of nivolumab plus ipilimumab. Pembrolizumab monotherapy can either be administered 200 mg every 3 weeks or 400 mg every 6 weeks over a 30-min intravenous infusion for the $1 \mathrm{~L}$ treatment of patients with metastatic NSCLC with TPS $\geq 1 \%$ until disease progression, unacceptable toxicity, or for a maximum of 24 months [1]. Pembrolizumab $200 \mathrm{mg}$ every 3 weeks was applied in the base case, to be consistent with the dosage used in the KEYNOTE-024 and -042 trials [5, 6]. The pembrolizumab dosage of $400 \mathrm{mg}$ every 6 weeks was tested in scenario analyses. Nivolumab dosage was $3 \mathrm{mg} / \mathrm{kg}$ every 2 weeks and ipilimumab was administered at a dose of $1 \mathrm{mg} / \mathrm{kg}$ every 6 weeks as a $1 \mathrm{~L}$ treatment for metastatic NSCLC with TPS $\geq$ $1 \%$ until disease progression, unacceptable toxicity, or for a maximum of 2 years [2].

\subsection{Time Horizon}

In CEAs of oncology treatments, it is commonplace to use a time horizon of sufficient duration to capture projected mortality for the vast majority of the modeled patient cohort. However, due to equivalent survival under the comparable efficacy assumption within the CMA, there were no differences in patient costs observed beyond the period of drug treatment. Therefore, the difference in cost between modeled strategies would remain constant from year 3 onwards, while absolute disease management costs continue to accrue at the same rate for each treatment over time. For this reason, we reported up to a 3-year time horizon.

The 3-year maximum time horizon was also approximately consistent with the follow-up within KEYNOTE-024, KEYNOTE-042, and CheckMate 227 Part 1a trial data used in the MAIC between therapies in the study by Halmos et al. [10].

\subsection{Survival Inputs}

We assumed comparable efficacy between pembrolizumab monotherapy and nivolumab in combination with 
ipilimumab for patients with PD-L1-positive metastatic NSCLC based on the findings of the MAIC study conducted by Halmos et al. [10].

PFS and OS Kaplan-Meier (KM) curves from the KEYNOTE-024 (data cut-off date of 15 February 2019) intention-to-treatment (ITT) population (i.e. patients with TPS $\geq 50 \%$ ) and KEYNOTE-042 (data cut-off date of 4 September 2018) TPS $\geq 50 \%$ and TPS $1-49 \%$ patient subgroups were extrapolated using a best-fitting piecewise approach based on the Akaike information criterion and the Bayesian information criterion. A parametric model was selected for the KEYNOTE-024 pembrolizumab arm OS extrapolation, given that no clear structural changes were observed from the KM curve and the cumulative hazard plot. A stepwise approach was used to pool data from KEYNOTE-024 and -042. First, the extrapolated PFS and OS of KEYNOTE-024 and KEYNOTE-042 patients with TPS $\geq 50 \%$ were combined, using sample sizes of the pembrolizumab arms in the two trials as weights, to generate the pooled PFS and OS for patients with TPS $\geq 50 \%$. Next, the combined PFS and OS for patients with TPS $\geq 50 \%$ treated with pembrolizumab and the PFS and OS for patients with TPS 1-49\% treated with pembrolizumab from KEYNOTE-042 were combined using the PD-L1 expression distribution in CheckMate 227 Part 1a as weights to generate PFS and OS for patients with TPS $\geq 1 \%$ treated with pembrolizumab. Based on the comparable efficacy assumption, the same PFS and OS were also used for patients treated with nivolumab plus ipilimumab.

Progressed disease survival (PDS) and death were calculated based on a three-state (i.e. progression-free [PF], progressed disease [PD], and death) partitioned survival model. PDS equaled OS minus PFS, and death equaled 1 minus OS. The length of the model cycle was 1 week.

\subsection{Treatment Duration Inputs}

In KEYNOTE-024, KEYNOTE-042, and CheckMate 227 Part 1a, patients could stop treatment before disease progression due to AEs [4-6]. Treatment duration was measured using time on treatment (ToT) in the base case, and treatment until disease progression was used as a scenario.

ToT KM curves from KEYNOTE-024 and KEYNOTE-042 were combined using the same weighted average method described above for patients with TPS $\geq 1 \%$ treated with pembrolizumab monotherapy.

Hellmann et al. [4] only reported a median treatment duration of 4.2 months for patients treated with nivolumab plus ipilimumab in CheckMate227 Part 1a. The authors did not include ToT KM curves in the publication, and thus Halmos et al. [10] were unable to estimate ToT HRs of pembrolizumab monotherapy versus nivolumab plus ipilimumab in their MAIC. While ToT could not be directly estimated via the MAIC, it was possible to approximate the impact of alignment of covariates between trials on ToT by evaluating how median PFS changed between the unadjusted value reported in CheckMate227 Partla and that estimated from the MAIC. The MAIC-based median PFS used in the model was 5.5 months, compared with a median PFS of 5.1 months observed in CheckMate227 Part 1a [4]. An adjusted median treatment duration for nivolumab plus ipilimumab was thus obtained by applying the ratio of the MAIC PFS to observed trial PFS (5.5/5.1) to the observed trial median ToT of 4.2 months, yielding an adjusted median treatment duration of 4.5 months. To generate a KM curve for patients who received nivolumab plus ipilimumab, an exponential interpolation of ToT was thus applied up to the 4.5 months of adjusted median treatment duration, and the subsequent ToT was generated through shifting PFS in parallel to reflect that changes in treatment discontinuations tend to slow over time.

Treatment durations were $\leq 2$ years for both pembrolizumab monotherapy and nivolumab plus ipilimumab based on the recommended dosing instructions in their respective FDA labels [1, 2, 21].

Figure 1 displays comparisons between the extrapolated PFS for patients treated with pembrolizumab or nivolumab plus ipilimumab, the weighted average ToT KMs for patients treated with pembrolizumab in KEYNOTE-024 and -042, and the simulated ToT for patients treated with nivolumab plus ipilimumab. The PFS curve does not overlap with the two ToT curves. The ToT curves are below the pooled PFS curve for most of the time, especially in the first 10 weeks, indicating a minority of patients discontinued treatment before progression due to AEs or other causes of treatment non-adherence.

\subsection{Adverse Event (AE) Inputs}

Hellmann et al. [4] reported TRAEs occurring in patients who received nivolumab plus ipilimumab in the CheckMate 227 Part 1a study. As TRAEs only account for a proportion of all-cause AEs, we instead extracted all-cause AEs occurring in $\geq 10 \%$ of patients receiving nivolumab plus ipilimumab in CheckMate 227 Part 1a from the nivolumab FDA label (updated in January 2021) [2]. For a fair comparison, we applied the same method to extract all-cause AEs for the pembrolizumab arm of KEYNOTE-042.

Given that the management of grade 3 or higher AEs is more likely to be resource-intensive [22], we only included grade 3 or higher all-cause AEs in our analysis (Table 1). Among AEs occurring in $\geq 10 \%$ of patients with either treatment, the incidence of grade 3 or higher AEs was lower with pembrolizumab monotherapy than with nivolumab plus ipilimumab. Patients treated with nivolumab plus ipilimumab had a $6 \%$ chance to develop grade 3 or higher fatigue/asthenia [2], and a $9 \%$ chance to develop grade 3 or higher hepatitis (including alanine aminotransferase [ALT] 
Fig. 1 Comparison of pembrolizumab/nivolumab + ipilimumab PFS versus pembrolizumab ToT and nivolumab plus ipilimumab ToT. $P F S$ progression-free survival, ToT time on treatment

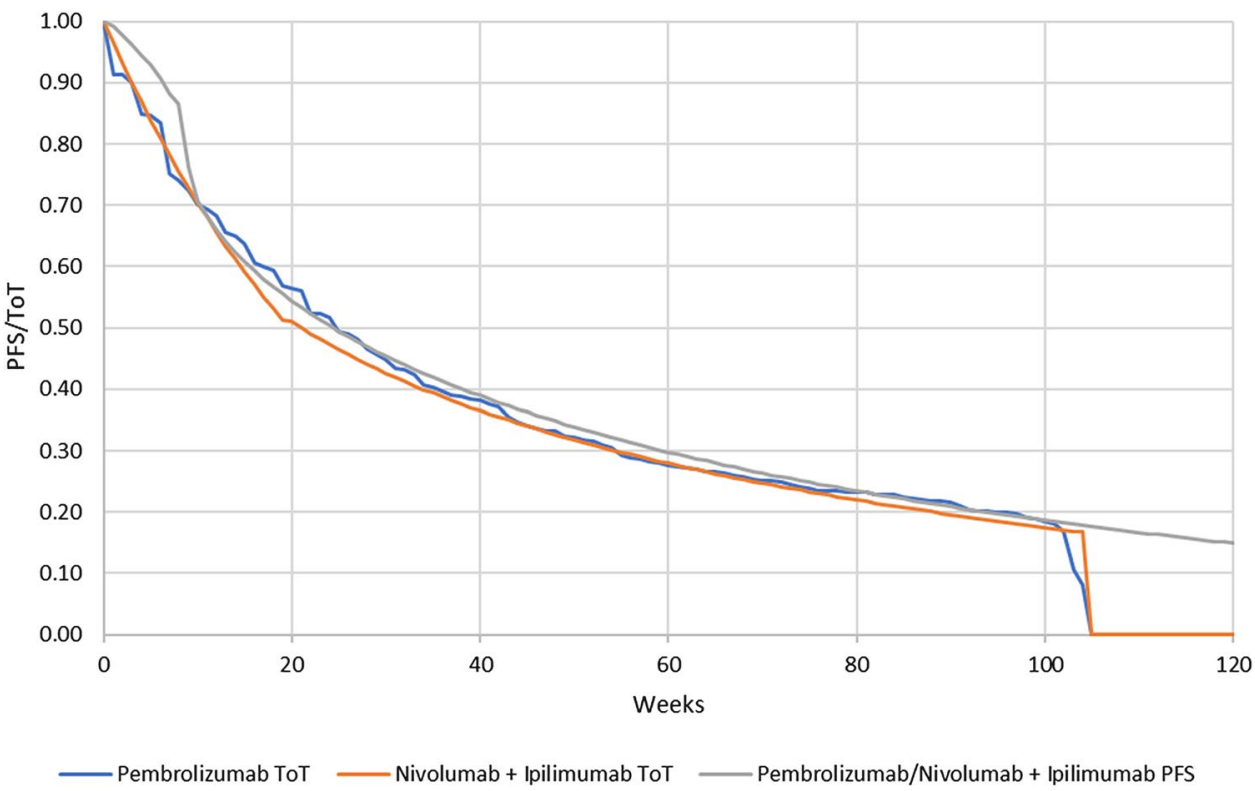

Table 1 Incidence and costs of grade 3 or higher AEs among AEs occurring in $\geq 10 \%$ of patients with either treatment

\begin{tabular}{llll}
\hline $\mathrm{AE}$ & Costs per episode & Incidence & \\
\cline { 3 - 4 } & & Pembrolizumab & $\begin{array}{l}\text { Nivolumab } \\
+ \text { ipili- } \\
\text { mumab }\end{array}$ \\
\hline Fatigue $^{\mathrm{a}}$ & $\$ 469$ & $3.1 \%$ & $6.0 \%$ \\
Hepatitis $^{\mathrm{b}}$ & $\$ 2512$ & $3.1 \%$ & $9.0 \%$ \\
Pneumonia $^{\mathrm{c}}$ & $\$ 4181$ & $8.5 \%$ & $7.0 \%$ \\
Total costs $^{\text {Pnet }}$ & & $\$ 448$ & $\$ 547$ \\
\hline
\end{tabular}

$A E$ adverse event, $A L T$ alanine transaminase, $A S T$ aspartate transaminase

${ }^{a}$ Includes fatigue and asthenia [2]

${ }^{\mathrm{b}}$ Includes ALT increased, AST increased, autoimmune hepatitis, blood bilirubin increased, hepatic enzyme increased, hepatic failure, hepatic function abnormal, hepatitis, hepatitis E, hepatocellular injury, hepatotoxicity, hyperbilirubinemia, immune-mediated hepatitis, liver function test abnormal, liver function test increased, transaminases increased [2]

${ }^{\mathrm{c}}$ Includes lower respiratory tract infection, lower respiratory tract infection bacterial, lung infection, pneumonia, pneumonia adenoviral, pneumonia aspiration, pneumonia bacterial, pneumonia Klebsiella, pneumonia influenza, pneumonia viral, atypical pneumonia, organizing pneumonia [2]

increased, aspartate aminotransferase [AST] increased, autoimmune hepatitis, blood bilirubin increased, hepatic enzyme increased, hepatic failure, hepatic function abnormal, hepatitis, hepatitis E, hepatocellular injury, hepatotoxicity, hyperbilirubinemia, immune-mediated hepatitis, liver function test abnormal, liver function test increased, transaminases increased [2]), whereas grade 3 or higher fatigue and hepatitis both had an incidence of $3.1 \%$ among patients treated with pembrolizumab. Patients treated with pembrolizumab had a slightly higher incidence of grade 3 or higher pneumonia (including lower respiratory tract infection, lower respiratory tract infection bacterial, lung infection, pneumonia, pneumonia adenoviral, pneumonia aspiration, pneumonia bacterial, pneumonia Klebsiella, pneumonia influenza, pneumonia viral, atypical pneumonia, organizing pneumonia [2]) compared with patients treated with nivolumab plus ipilimumab ( $8.5 \%$ vs. $7.0 \%)$.

Given that the AE incidence inputs were unadjusted for differences in patient baseline characteristics between KEYNOTE-042 and CheckMate 227 Part 1a, we conducted scenario analyses by increasing and decreasing estimated $\mathrm{AE}$ costs by $10 \%$.

\subsection{Cost Inputs}

As the CMA was from a US payer perspective, a $20 \%$ patient co-insurance rate was applied [23]. All costs were discounted at 3\% annually [24] and were inflated to 2020 US dollars using the Medical Care component of the US Consumer Price Index [25].

\subsubsection{Drug Acquisition and Administration Costs}

Wholesale acquisition costs (WACs) extracted from the First Databank (FDB) MedKnowledge Drug Database were used as unit costs for each drug [26]. Vial sharing was not allowed to reflect US clinical practice. Drug acquisition costs per cycle were calculated based on administration instructions in FDA labels $[1,2,21]$ and were then applied to patients' treatment durations. 
Unit drug administration costs were extracted from the KEYNOTE-042 CEA published by Huang et al. [14]. They were multiplied by the administration time indicated from the FDA labels $[1,2,21]$ and were then applied to patients' treatment durations.

\subsubsection{Subsequent Treatment Costs}

The percentage of pembrolizumab-treated patients who received subsequent lines of therapy after treatment discontinuation and distribution across subsequent therapies were estimated from KEYNOTE-042 trial data. Subsequent treatment for patients treated with nivolumab plus ipilimumab was retrieved from the study by Hellmann et al. [4]. In that publication, subsequent therapies were presented by treatment arm in the PD-L1 TPS $\geq 1 \%$ population for individual subsequent immunotherapy drugs and overall subsequent chemotherapy and overall subsequent targeted therapy [4]. Individual chemotherapy and individual targeted therapy distributions were assumed to be the same as distributions observed in patients treated with pembrolizumab in KEYNOTE-042. Average treatment durations of subsequent anti-PD-1/PD-L1 treatments and chemotherapy/targeted therapy treatments were estimated from KEYNOTE-042.

Drug acquisition, administration, and premedication costs of each subsequent treatment were multiplied by subsequent treatment distributions and durations and were applied to discontinued patients in each model cycle to estimate total subsequent treatment costs.

We acknowledge that differences in subsequent treatments used in the two trials might be attributable to differences in the periods when the trials were conducted, the geographic distribution of patients and associated medical guidelines and physician preferences. We conducted a scenario analysis by assuming patients treated with nivolumab plus ipilimumab shared the same subsequent treatment costs as patients treated with pembrolizumab.

\subsubsection{AE Management Costs}

Grade 3 or higher all-cause AE management costs included both hospitalization and physician visit costs. The proportion of patients hospitalized for AE management were estimated from KEYNOTE-042. Hospitalization costs for each AE were extracted from Medicare feefor-service payments [27]. The remaining patients were assumed to incur the cost of a physician visit, which was based on Center for Medicare and Medicaid Services costs for a hospital outpatient visit, extracted from the study by Huang et al. [14].

\subsubsection{Disease Management and Terminal Care Costs}

Average weekly non-drug disease management costs by health states and one-time terminal care costs were extracted from the study by Huang et al. [14]. Within that study, both costs were estimated from 2013 Surveillance, Epidemiology, and End Results-Medicare metastatic NSCLC data. Disease management costs included inpatient, outpatient, emergency room, skilled nursing facility, and hospice costs, while terminal care costs included inpatient, palliative chemotherapy, physician consultation, laboratory and diagnostic test, and hospice costs occurring in the last 30 days of life [14]. These costs were assumed to be the same across comparators.

Annual disease management costs were applied to PFS and PDS to estimate total costs spent on disease management in the PF and PD health states. Terminal care costs were applied to patients who died.

Key model inputs are presented in Table 2.

\subsection{Scenario Analyses}

Scenario analyses were conducted for pembrolizumab dosage, cost discount rate, predicted PFS, treatment durations of pembrolizumab and nivolumab plus ipilimumab, subsequent treatment costs, $\mathrm{AE}$ cost, disease management cost, and terminal care cost to address the uncertainty surrounding these parameters. Results and tornado diagrams were presented by time horizon.

A probabilistic sensitivity analysis (PSA) was performed using 1000 iterations to test parameter uncertainty. Means and frequency distribution of cost savings were reported by time horizon. Parameter distributions used in the PSA are reported in Table 3.

\section{Results}

\subsection{Base-Case Results}

Table 4 presents base-case results for patients with TPS $\geq$ $1 \%$ metastatic NSCLC. Pembrolizumab generated $\$ 54,343$, $\$ 75,744$, and $\$ 76,259$ per patient cost savings compared with nivolumab plus ipilimumab within 1,2, and 3 years, respectively. As the maximum treatment durations for both pembrolizumab and nivolumab plus ipilimumab are 2 years, the difference in costs savings between years 2 and 3 came from subsequent therapy costs that were applied following the last administration of pembrolizumab or nivolumab plus ipilimumab.

Most of the cost savings were attributable to drug acquisition costs, with the remainder distributed across drug 
Table 2 Key model inputs

\begin{tabular}{|c|c|c|}
\hline Parameter & Value & Source(s) \\
\hline \multicolumn{3}{|l|}{ PD-L1 distribution } \\
\hline TPS $\geq 50 \%$ & $51.8 \%$ & \multirow[t]{2}{*}{ Hellmann et al. [4] } \\
\hline TPS $1-49 \%$ & $48.2 \%$ & \\
\hline \multicolumn{3}{|c|}{$\begin{array}{l}\text { Patient source in pooled } \\
\text { KN024/042 TPS } \geq 50 \% \text { patients }\end{array}$} \\
\hline KN024 & $34.0 \%$ & \multirow[t]{2}{*}{$\mathrm{KN} 024^{\mathrm{a}}$ and $\mathrm{KN} 042^{\mathrm{b}}$} \\
\hline KN042 & $66.0 \%$ & \\
\hline \multicolumn{3}{|l|}{ Pembrolizumab } \\
\hline PFS & $\begin{array}{l}\text { KN024 KM to week 10, generalized gamma there- } \\
\text { after; KN042 KM to week 9, Weibull thereafter }\end{array}$ & \multirow[t]{7}{*}{$\mathrm{KN} 024^{\mathrm{a}}$ and $\mathrm{KN} 042^{\mathrm{b}}$} \\
\hline OS & $\begin{array}{l}\text { KN024 log-logistic from week } 0 \text { to year } 4 ; \mathrm{KN} 042 \\
\text { TPS } \geq 50 \% \text { to week } 30 \text {, TPS } 1-49 \% \text { to week } 46 \text {, } \\
\text { exponential thereafter }\end{array}$ & \\
\hline ToT & KN024/042 ToT KM, capped at 2 years & \\
\hline \multicolumn{2}{|l|}{ Nivolumab + ipilimumab } & \\
\hline PFS & Same as pembrolizumab PFS & \\
\hline OS & Same as pembrolizumab OS & \\
\hline ToT & $\begin{array}{l}\text { Exponential interpolation until median ToT, } \\
\text { adjusted by relative change in PFS afterwards, } \\
\text { capped at } 2 \text { years }\end{array}$ & \\
\hline \multicolumn{3}{|l|}{ Treatment intensity } \\
\hline Pembrolizumab & $98.1 \%$ & $\mathrm{KN} 024^{\mathrm{a}}$ and $\mathrm{KN} 042^{\mathrm{b}}$ \\
\hline Nivolumab & $98.2 \%$ & \multirow[t]{2}{*}{ Hellmann et al. [4] } \\
\hline Ipilimumab & $100 \%$ & \\
\hline \multicolumn{3}{|l|}{ Drug price } \\
\hline Pembrolizumab & $\$ 4862.04 / 100 \mathrm{mg}$ vial & \multirow{3}{*}{$\begin{array}{l}\text { First Databank MedKnowledge Drug Database WAC } \\
\text { [26] }\end{array}$} \\
\hline Nivolumab & $\begin{array}{l}\$ 1096.74 / 40 \mathrm{mg} \text { vial } \\
\$ 2741.85 / 100 \mathrm{mg} \text { vial } \\
\$ 6580.45 / 240 \mathrm{mg} \text { vial }\end{array}$ & \\
\hline Ipilimumab & $\begin{array}{l}\$ 7501.41 / 50 \mathrm{mg} \text { vial } \\
\$ 30,005.56 / 200 \mathrm{mg} \text { vial }\end{array}$ & \\
\hline \multicolumn{3}{|l|}{ Administration costs (hourly) } \\
\hline First-hour IV infusion & $\$ 119.97$ & \multirow[t]{3}{*}{ Huang et al. [14] } \\
\hline Additional-hour IV infusion & $\$ 26.08$ & \\
\hline Subsequent IV infusion & $\$ 57.37$ & \\
\hline \multicolumn{3}{|c|}{ Disease management costs (weekly) } \\
\hline \multicolumn{3}{|c|}{$\mathrm{PF}$} \\
\hline Year 1 & $\$ 1344$ & \multirow[t]{5}{*}{ Huang et al. [14] } \\
\hline Year 2 & $\$ 539$ & \\
\hline Year 3 & $\$ 413$ & \\
\hline Years 4-5 & $\$ 304$ & \\
\hline Years 6+ & $\$ 212$ & \\
\hline \multicolumn{3}{|l|}{$\mathrm{PD}$} \\
\hline Year 1 & $\$ 1329$ & \multirow[t]{5}{*}{ Huang et al. [14] } \\
\hline Year 2 & $\$ 1034$ & \\
\hline Year 3 & $\$ 854$ & \\
\hline Years 4-5 & $\$ 726$ & \\
\hline Years 6+ & $\$ 767$ & \\
\hline Terminal care costs & $\$ 17,289$ & Huang et al. [14] \\
\hline \multicolumn{3}{|l|}{ Subsequent treatment costs } \\
\hline Pembrolizumab & $\$ 10,582$ & $\begin{array}{l}\text { Based on subsequent treatment distribution in } \\
\mathrm{KN} 042^{\mathrm{b}}\end{array}$ \\
\hline
\end{tabular}


Table 2 (continued)

\begin{tabular}{lll}
\hline Parameter & Value & Source(s) \\
\hline Nivolumab + ipilimumab & $\$ 11,711$ & $\begin{array}{c}\text { Based on subsequent treatment distribution reported } \\
\text { by Hellmann et al. [4] }\end{array}$ \\
$\begin{array}{l}\text { AE costs } \\
\text { Pembrolizumab }\end{array}$ & $\$ 448$ & $\begin{array}{l}\text { Based on AE incidence estimated from KN042 } \\
\text { Nivolumab + ipilimumab }\end{array}$ \\
\end{tabular}

$A E$ adverse event, $I V$ intravenous, $K M$ Kaplan-Meier, $K N$ KEYNOTE, $O S$ overall survival, $P D-L 1$ programmed death-ligand $1, P F$ progressionfree, $P F S$ progression-free survival, $P D$ progressed disease, ToT time on treatment, $T P S$ tumor proportion score, WAC wholesale acquisition cost ${ }^{a}$ Data cut-off date of 15 February 2019

${ }^{\mathrm{b}}$ Data cut-off date of 4 September 2018

Table 3 Probabilistic sensitivity analysis distributions

\begin{tabular}{ll}
\hline Model parameter & PSA distribution \\
\hline $\begin{array}{l}\text { Pembrolizumab PFS extrapolation model } \\
\text { parameters }\end{array}$ & Multivariate normal distribution \\
$\begin{array}{l}\text { Pembrolizumab OS extrapolation model } \\
\text { parameters }\end{array}$ & Multivariate normal distribution \\
Drug administration costs & Log-normal distribution with 20\% of base-case mean as SE \\
Disease management costs & Log-normal distribution with 20\% of base-case mean as SE \\
Terminal care costs & Log-normal distribution with 20\% of base-case mean as SE \\
AE costs & Log-normal distribution with 20\% of base-case mean as SE \\
\hline
\end{tabular}

$A E$ adverse event, $O S$ overall survival, $P F S$ progression-free survival, $P S A$ probabilistic sensitivity analysis, $S E$ standard error

Table 4 Base-case results, by number of years of follow-up

\begin{tabular}{|c|c|c|c|c|c|c|c|c|c|}
\hline \multirow[t]{2}{*}{ Cost category } & \multicolumn{3}{|l|}{1 year } & \multicolumn{3}{|l|}{2 years } & \multicolumn{3}{|l|}{3 years } \\
\hline & $\mathrm{P}$ & NI & $\mathrm{d}($ Costs $)$ & $\mathrm{P}$ & NI & $\mathrm{d}($ Costs $)$ & $\mathrm{P}$ & NI & $\mathrm{d}($ Costs $)$ \\
\hline Drug acquisition & $\$ 74,068$ & $\$ 126,828$ & $-\$ 52,760$ & $\$ 103,492$ & $\$ 177,635$ & $-\$ 74,143$ & $\$ 103,492$ & $\$ 177,635$ & $-\$ 74,143$ \\
\hline Drug administration & $\$ 914$ & $\$ 1543$ & $-\$ 629$ & $\$ 1277$ & $\$ 2155$ & $-\$ 878$ & $\$ 1277$ & $\$ 2155$ & $-\$ 878$ \\
\hline Subsequent treatment & $\$ 7159$ & $\$ 8013$ & $-\$ 854$ & $\$ 9905$ & $\$ 10,528$ & $-\$ 623$ & $\$ 10,302$ & $\$ 11,440$ & $-\$ 1138$ \\
\hline Disease management & $\$ 53,750$ & $\$ 53,750$ & $\$ 0$ & $\$ 73,262$ & $\$ 73,262$ & $\$ 0$ & $\$ 84,629$ & $\$ 84,629$ & $\$ 0$ \\
\hline Terminal care & $\$ 7095$ & $\$ 7095$ & $\$ 0$ & $\$ 10,179$ & $\$ 10,179$ & $\$ 0$ & $\$ 12,150$ & $\$ 12,150$ & $\$ 0$ \\
\hline AEs & $\$ 448$ & $\$ 547$ & $-\$ 99$ & $\$ 448$ & $\$ 547$ & $-\$ 99$ & $\$ 448$ & $\$ 547$ & $-\$ 99$ \\
\hline Total costs & $\$ 143,434$ & $\$ 197,776$ & $-\$ 54,343$ & $\$ 198,563$ & $\$ 274,307$ & $-\$ 75,744$ & $\$ 212,298$ & $\$ 288,557$ & $-\$ 76,259$ \\
\hline
\end{tabular}

$A E$ adverse event, $d($ Costs) incremental costs, $P$ pembrolizumab, NI nivolumab plus ipilimumab

administration, subsequent treatment, and AE management costs.

The study assumed comparable treatment efficacy across arms and the same unit disease management costs by health state and terminal care costs across treatments, and therefore incremental disease management costs and terminal care costs were 0 .

\subsection{Sensitivity Analyses Results}

Table 5 displays the results of the scenario analyses.

If patients receiving pembrolizumab switched from the $200 \mathrm{mg}$ every 3 weeks dose to the $400 \mathrm{mg}$ every 6 weeks dose, per patient cost savings reduced to $\$ 52,078, \$ 72,097$, and $\$ 72,612$ within 1,2 , and 3 years, respectively. The reduction was caused by the 24 -month maximum treatment 
Table 5 Scenario analyses results

\begin{tabular}{|c|c|c|c|}
\hline \multirow[t]{2}{*}{ Scenario } & \multicolumn{3}{|c|}{$\begin{array}{l}\mathrm{d} \text { (Costs) for pembrolizumab vs. nivolumab }+ \\
\text { ipilimumab }\end{array}$} \\
\hline & 1 year & 2 years & 3 years \\
\hline Base case incremental costs & $-\$ 54,343$ & $-\$ 75,744$ & $-\$ 76,259$ \\
\hline \multicolumn{4}{|l|}{ Pembrolizumab dosage } \\
\hline $400 \mathrm{mg}$ every 6 weeks & $-\$ 52,078$ & $-\$ 72,097$ & $-\$ 72,612$ \\
\hline \multicolumn{4}{|l|}{ Cost discount rate } \\
\hline $0 \%$ & $-\$ 54,343$ & $-\$ 76,386$ & $-\$ 76,932$ \\
\hline $5 \%$ & $-\$ 54,343$ & $-\$ 75,336$ & $-\$ 75,832$ \\
\hline \multicolumn{4}{|l|}{ Pembrolizumab PFS } \\
\hline $\begin{array}{l}\text { KN024 KM to week 10, exponential thereafter; KN042 } \\
\text { KM to week 9, generalized gamma thereafter }\end{array}$ & $-\$ 53,960$ & $-\$ 73,778$ & $-\$ 74,257$ \\
\hline \multicolumn{4}{|l|}{ Treatment duration } \\
\hline Time to progression & $-\$ 59,714$ & $-\$ 83,851$ & $-\$ 83,949$ \\
\hline \multicolumn{4}{|l|}{ Subsequent treatment costs } \\
\hline Same costs & $-\$ 53,488$ & $-\$ 75,121$ & $-\$ 75,121$ \\
\hline$+10 \%$ & $-\$ 54,427$ & $-\$ 75,806$ & $-\$ 76,373$ \\
\hline$-10 \%$ & $-\$ 54,257$ & $-\$ 75,682$ & $-\$ 76,145$ \\
\hline \multicolumn{4}{|l|}{ AE costs } \\
\hline$+10 \%$ & $-\$ 54,352$ & $-\$ 75,754$ & $-\$ 76,269$ \\
\hline$-10 \%$ & $-\$ 54,332$ & $-\$ 75,734$ & $-\$ 76,249$ \\
\hline \multicolumn{4}{|l|}{ Disease management costs } \\
\hline Nivolumab + ipilimumab $+5 \%$ & $-\$ 57,030$ & $-\$ 79,407$ & $-\$ 80,490$ \\
\hline Nivolumab + ipilimumab $-5 \%$ & $-\$ 51,655$ & $-\$ 72,081$ & $-\$ 72,028$ \\
\hline \multicolumn{4}{|l|}{ Terminal care costs } \\
\hline Nivolumab + ipilimumab $+5 \%$ & $-\$ 54,697$ & $-\$ 76,253$ & $-\$ 76,867$ \\
\hline Nivolumab + ipilimumab $-5 \%$ & $-\$ 53,987$ & $-\$ 75,235$ & $-\$ 75,652$ \\
\hline
\end{tabular}

$A E$ adverse event, $d($ Costs) incremental costs, KM Kaplan-Meier, KN KEYNOTE, PFS progression-free survival duration suggested in pembrolizumab's FDA label [1]. The $200 \mathrm{mg}$ per 3 weeks dosage led to 35-cycle administration within 24 months, while the $400 \mathrm{mg}$ per 6 weeks dosage led to 18 -cycle administration within 24 months. Although the number of administrations was reduced by 17 cycles, drug acquisition costs were increased by 1 cycle of $200 \mathrm{mg}$ pembrolizumab costs, thereby reducing the overall cost savings.

Changing the annual cost discount rate only affected incremental cost estimation within 2 years and 3 years. No cost discount led to more cost savings and a $5 \%$ cost discount rate led to fewer cost savings.

OS only affected disease management and terminal care costs and had no influence on incremental total costs, and thus was not tested in the scenario analysis.

Predicted PFS was tested by applying the second-best distribution for extrapolation, i.e. extrapolation based on exponential distribution after week 10 for KEYNOTE-024 PFS KM, and generalized gamma distribution after week 9 for KEYNOTE-042 PFS KM. Cost savings changed because treatment duration for nivolumab plus ipilimumab was partly constructed by applying a ratio to its PFS.

Using PFS as a proxy for treatment duration greatly increased pembrolizumab's cost savings, as the higher the proportion of patients on treatment, the larger the differences in disease treatment costs.

Cost savings estimated from the scenario analyses are also displayed in Fig. 2.

The mean cost savings of pembrolizumab versus nivolumab plus ipilimumab estimated from the PSA were $\$ 59,585, \$ 85,292$, and $\$ 85,859$, respectively. Figure 3 displays the frequency distribution of the cost savings.

\section{Discussion}

The results of the study show that with the assumption of comparable treatment efficacy, $1 \mathrm{~L}$ pembrolizumab generates significant cost savings compared with nivolumab in combination with ipilimumab for the treatment of patients with 
Fig. 2 Tornado diagram for scenario analyses of the costminimization analysis. The second-best distribution used for PFS extrapolation in the scenario analyses refers to $\mathrm{KN} 024$ $\mathrm{KM}$ to week 10, exponential thereafter; and KN042 KM to week 9, generalized gamma thereafter. $A E$ adverse event, $K N$ KEYNOTE, KM Kaplan-Meier, $N I$ nivolumab plus ipilimumab, $P F S$ progression-free survival, $Q 6 W$ every 6 weeks
Time horizon - 1 years

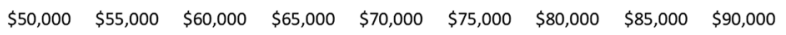

Pembrolizumab dosage 400mg Q6W

Cost discount $0 \% / 5 \%$

PFS extrapolated by second best distribution

Treat until progression

Same subsequent treatment costs

Subsequent treatment costs $-10 \% /+10 \%$

AE costs $-10 \% /+10 \%$

NI disease management costs $-5 \% /+5 \%$

$\mathrm{NI}$ terminal care costs $-5 \% /+5 \%$
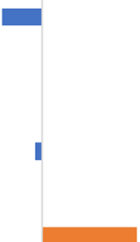

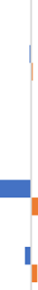

Time horizon - 2 years

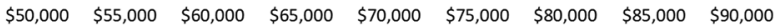

Pembrolizumab dosage 400mg Q6W

Cost discount $0 \% / 5 \%$

PFS extrapolated by second best distribution

Treat until progression

Same subsequent treatment costs

Subsequent treatment costs $-10 \% /+10 \%$

AE costs $-10 \% /+10 \%$

$\mathrm{NI}$ disease management costs $-5 \% /+5 \%$

$\mathrm{NI}$ terminal care costs $-5 \% /+5 \%$

Time horizon -3 years

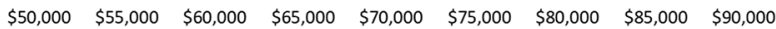

Pembrolizumab dosage 400mg Q6W

Cost discount $0 \% / 5 \%$

PFS extrapolated by second best distribution

Treat until progression

Same subsequent treatment costs

Subsequent treatment costs $-10 \% /+10 \%$

AE costs $-10 \% /+10 \%$

NI disease management costs $-5 \% /+5 \%$

NI terminal care costs $-5 \% /+5 \%$

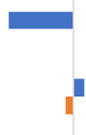

1

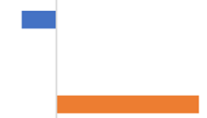

【

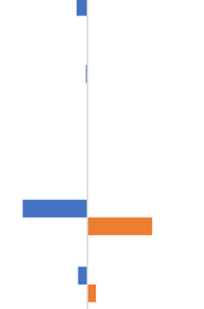

(1)

$\%$

\section{.}

.

\section{. $355,000+\$ 60,000$ years}


Fig. 3 Frequency distribution of pembrolizumab versus nivolumab plus ipilimumab cost savings from 1000 iterations of the probabilistic sensitivity analysis
Time horizon - 1 year

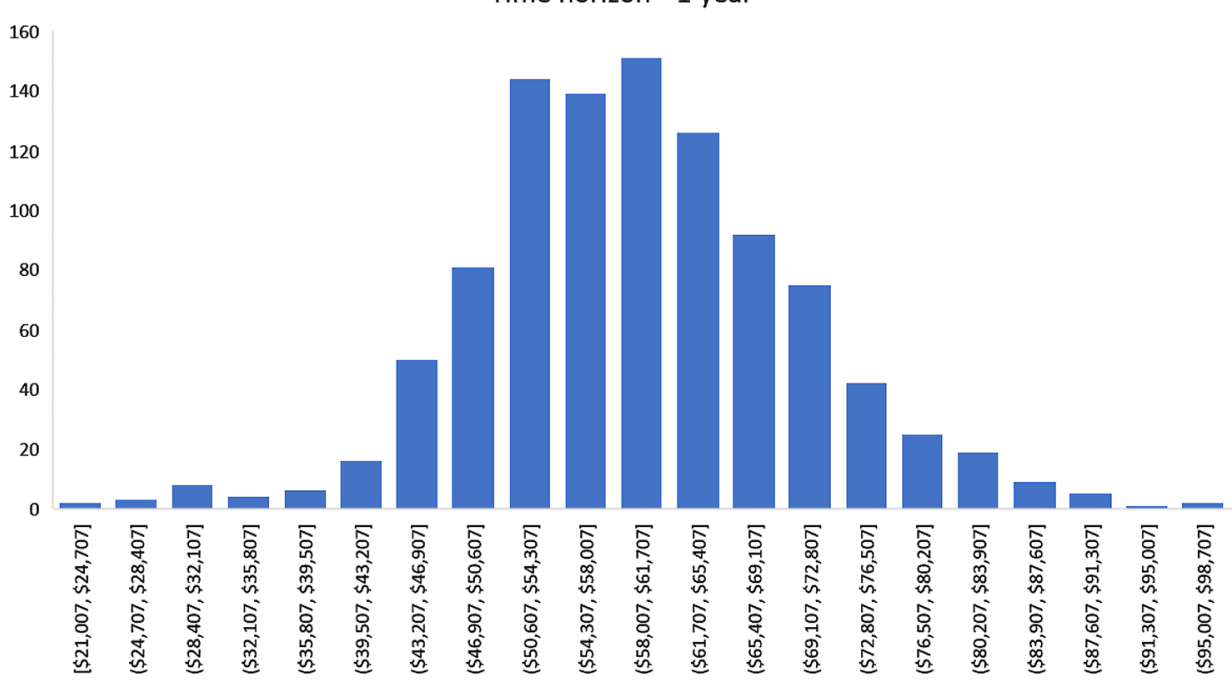

Time horizon -2 years

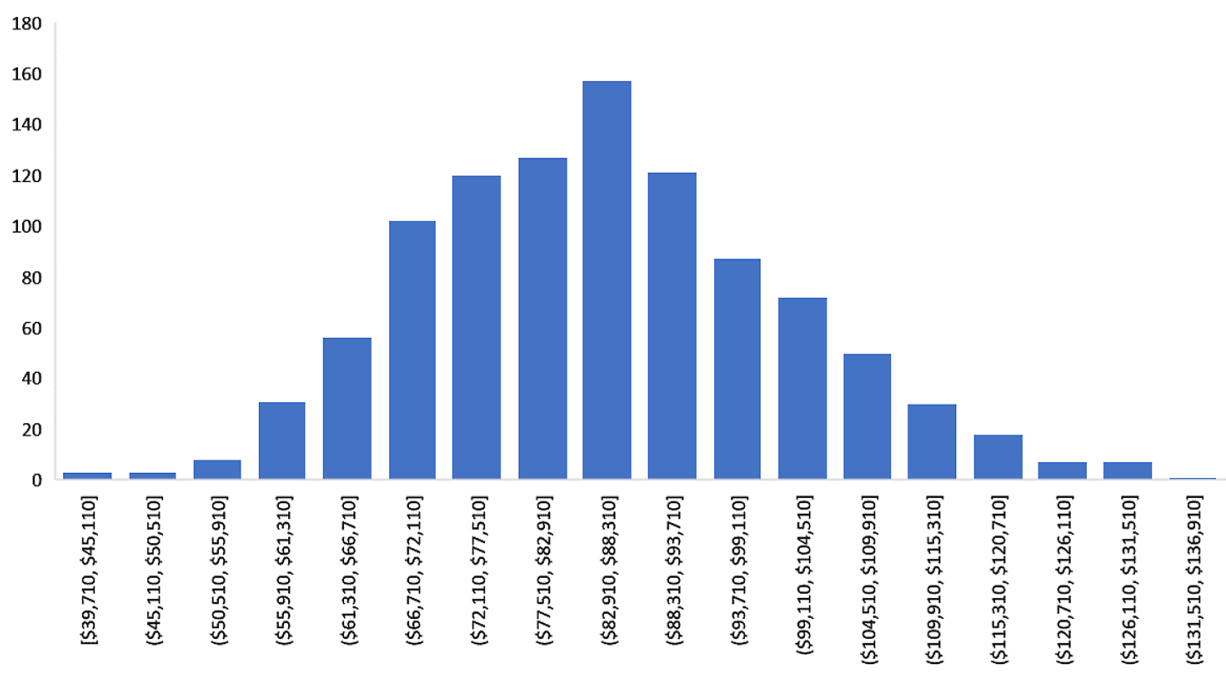

Time horizon -3 years

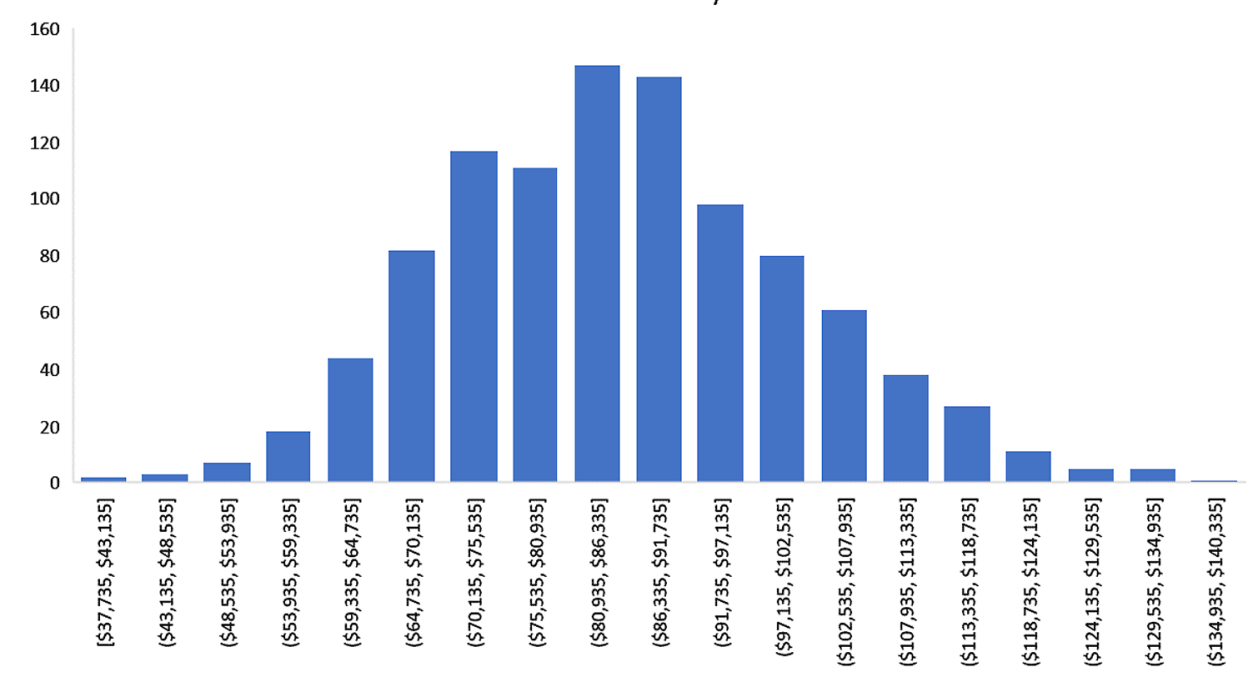


metastatic, PD-L1-positive NSCLC within the first 3 years. The findings are mainly driven by greater drug acquisition costs associated with higher unit costs of nivolumab and ipilimumab compared with pembrolizumab monotherapy. The findings are robust over different scenarios.

This study has several limitations. First, the US has a segmented healthcare system, with Medicare covering people aged 65 years and above as well as those with a longterm disability, Medicaid covering people with low income, and different private insurance companies covering most of the remainder. Each payer has their own payment rates. In general, payment rates are higher in private insurance than Medicare and Medicaid [28, 29]. Due to data limitation, we were unable to obtain costs for different types of payers. Most cost inputs used in the CMA were based on Medicare payment rates, except drug prices, which were based on WACs. With acknowledgment that a fairly large proportion of patients in KEYNOTE-024, -042, and CheckMate 227 were under age 65 years [4-6], we would rather use Medicare payment rates as a proxy for the average costs across different payer types, and vary these costs in the scenario analyses. Pembrolizumab's large cost savings remain across these scenarios.

Second, the underlying assumption that pembrolizumab monotherapy has comparable OS and PFS as nivolumab plus ipilimumab is based on an anchored MAIC, rather than a head-to-head RCT. Although RCTs are the gold standard to measure comparative efficacy [30], MAICs have the strength of adjusting for between-trial differences in patient baseline characteristics to enhance analytical validity, making them superior to traditional ITC methods, such as NMAs [31]. In addition, as RCTs are expensive and time-consuming, MAICs offer a less costly and timely option for reliable treatment efficacy comparison across trials [32]. We acknowledge that MAIC is based on the assumption that no unobserved factors may confound outcome comparisons [32]. The large set of patient baseline characteristics controlled for by Halmos et al. [10] in the MAIC is anticipated to limit confounding effects from missing variables. Another limitation of the MAIC study by Halmos et al. [10] was that AE incidences were not compared because of the limited $\mathrm{AE}$ data presented in the CheckMate 227 Part 1a trial publication. Based on the experience of working on other metastatic NSCLC CEAs, AEs contribute minimally to overall patient utilities and therefore we do not consider this to be a major concern. For example, in the CEA based on KEYNOTE-024 conducted by Huang et al. [13] utility values for each timeto-death category cross treatment arms were found to be not significantly different, despite incidences of grade 3 or higher all-cause AEs in the pembrolizumab arm being much lower compared with the chemotherapy arm, except pneumonitis. We limited the time horizon of the study to 3 years, which was approximately the same as the follow-up period of the trial by Halmos et al. [10]. In the absence of longerterm comparable efficacy evidence, any generalization of the study findings beyond the maximum 3-year time horizon should be done with caution.

Third, the CheckMate 227 Part 1a trial publication did not report ToT KMs [4]. The ToT of nivolumab in combination with ipilimumab was thus simulated based on an adjusted median treatment duration incorporating observed differences between MAIC-based median PFS for the combination in the model and the median PFS derived from the CheckMate 227 Part 1a trial [4], and assumptions of an exponential distribution before the median treatment duration and a fixed ratio with PFS afterwards. The simulated ToT curve for nivolumab plus ipilimumab only had slight differences from the ToT of pembrolizumab in patients with TPS $\geq 1 \%$, and therefore the approach did not put nivolumab plus ipilimumab at a disadvantage for cost estimation.

Fourth, due to a lack of access to detailed CheckMate 227 Part 1a all-cause AE data, the study extracted grade 3 or higher all-cause AEs among AEs occurring in $\geq 10 \%$ of patients for nivolumab plus ipilimumab from nivolumab's FDA label, and applied the same criteria to the KEYNOTE-042 data to estimate grade 3 or higher all-cause AE inputs for pembrolizumab monotherapy. The approach could not capture grade 3 or higher AEs with a lower incidence, and likely underestimated $\mathrm{AE}$ costs for both regimens.

Fifth, the KEYNOTE-042 trial included 12\% of patients who had non-metastatic advanced NSCLC, but the CheckMate 227 trial did not report the proportion of patients with non-metastatic recurrent NSCLC $[4,5]$. In the event that a dramatically different proportion of patients with metastatic disease was present in CheckMate 227 compared with KEYNOTE-042, model inputs such as subsequent treatment and AEs may reflect patients with different disease stage distributions across treatments. In this study, both subsequent treatment and $\mathrm{AE}$ cost estimation was based on unadjusted inputs, which are limitations that we are not able to overcome. Both costs were tested in the scenario analyses by applying a $10 \%$ increase or decrease, and subsequent treatment costs were also tested by assuming nivolumab plus ipilimumab shared the same costs as pembrolizumab. The large cost savings of pembrolizumab remained.

Finally, the study assumed the same disease management and terminal care costs for patients with either treatment. Weekly disease management costs from the study by Huang et al. [14] were defined as costs generated from inpatient, emergency care, outpatient, skilled nursing facility and hospice visits other than anticancer treatment. Based on this definition, it is reasonable to assume disease management costs do not vary by treatment based on assumed comparable efficacy. Scenario analyses were conducted by setting management costs and terminal care costs for patients treated with 
nivolumab in combination with ipilimumab $-5 \%$ lower or + $5 \%$ higher than patients treated with pembrolizumab. Results show that pembrolizumab's large cost savings remained in these scenarios.

This study is the first economic evaluation of $1 \mathrm{~L}$ pembrolizumab versus nivolumab plus ipilimumab for patients with metastatic PD-L1-positive NSCLC without EGFR or $A L K$ genomic tumor aberrations. Often, a CEA instead of a CMA is used to compare both the effectiveness and costs of two treatments. In the present analysis, given the comparable efficacy findings between treatments reported by Halmos et al. [10], a CEA would add little value. The minimal difference in treatment effects between the two treatments tends to yield very small nominal differences in effectiveness, and ICERs are likely to be extremely large, which might lead to mis- or over-interpretation of results. In contrast, results from a CMA, which explicitly reflect the lack of evidence of differential treatment effects, are more straightforward for payers to interpret and more actionable.

\section{Conclusion}

The findings of the study imply pembrolizumab monotherapy can generate substantial cost savings compared with nivolumab plus ipilimumab therapy for patients with metastatic PD-L1-positive NSCLC, for US payers in the short term. In addition, Boyer et al. reported that adding ipilimumab to pembrolizumab does not improve pembrolizumab's efficacy but leads to a higher incidence of grade 3 or higher AEs as $1 \mathrm{~L}$ treatment for patients with metastatic NSCLC with TPS $\geq 50 \%$ without $E G F R$ or $A L K$ aberrations, based on the randomized, double-blind, phase III KEYNOTE-598 study [33]. These findings raise new questions regarding the clinical benefits of combining ipilimumab with an anti-PD-1 when treating metastatic NSCLC.

Acknowledgements The authors thank Dr Ina Nikolaeva (Merck \& Co., Inc., Kenilworth, NJ, USA) for her copyediting and formatting assistance, and Chrysostomos Kalyvas (Merck Sharp \& Dohme Corp., a subsidiary of Merck \& Co., Inc., Kenilworth, NJ, USA) for reviewing and revising the statistical methodology description in the introduction and proofreading the remainder of the manuscript.

\section{Declarations}

Funding This research was supported by Merck \& Co., Inc., Kenilworth, NJ, USA.

Conflicts of Interest Nan Qiao, Ralph Insinga, and Thomas Burke are employees of Merck \& Co., Inc., Kenilworth, NJ, USA. Gilberto de Lima Lopes Junior is an employee of the University of Miami Clinical Oncology Sector, which received research grants from Merck \& Co., Inc., Kenilworth, NJ, USA.
Ethics Approval Not applicable.

Consent to Participate Not applicable.

Consent for Publication Not applicable.

Availability of Data and Material Most model inputs and their sources are included in this paper. Patient-level data collected in KEYNOTE-024 and -042 are not available. The authors will assist with any reasonable replication attempts for 2 years following publication.

Code Availability Not available. The authors will assist with any reasonable replication attempts for 2 years following publication.

Author Contributions Nan Qiao, Ralph Insinga, and Thomas Burke developed the study concept, and designed and planned the study. Nan Qiao built the cost-minimization model, conducted analyses, and drafted the manuscript. Ralph Insinga and Thomas Burke provided data inputs to the model. Gilberto de Lima Lopes Junior planned the study. All authors interpreted the results, critically reviewed and/or revised the manuscript, approved the content of the manuscript and its submission, and agreed to be accountable for all aspects of the manuscript.

Open Access This article is licensed under a Creative Commons Attribution-NonCommercial 4.0 International License, which permits any non-commercial use, sharing, adaptation, distribution and reproduction in any medium or format, as long as you give appropriate credit to the original author(s) and the source, provide a link to the Creative Commons licence, and indicate if changes were made. The images or other third party material in this article are included in the article's Creative Commons licence, unless indicated otherwise in a credit line to the material. If material is not included in the article's Creative Commons licence and your intended use is not permitted by statutory regulation or exceeds the permitted use, you will need to obtain permission directly from the copyright holder. To view a copy of this licence, visit http://creativecommons.org/licenses/by-nc/4.0/.

\section{References}

1. US FDA. KEYTRUDA® (pembrolizumab) injection, for intravenous use. https://www.accessdata.fda.gov/drugsatfda_docs/label/ 2020/125514s088lbl.pdf. Accessed 4 Mar 2021

2. US FDA. OPDIVO (nivolumab) injection, for intravenous use 2021. https://www.accessdata.fda.gov/drugsatfda_docs/label/ 2021/125554s090lbl.pdf. Accessed 4 Mar 2021

3. National Comprehensive Cancer Network. NCCN clinical practice guidelines in oncology-non-small cell lung cancer, version 2.2021. 2020. https://www.nccn.org/professionals/physician_gls/ pdf/nscl_blocks.pdf. Accessed 28 Jan 2021

4. Hellmann MD, Paz-Ares L, Bernabe Caro R, et al. Nivolumab plus ipilimumab in advanced non-small-cell lung cancer. N Engl J Med. 2019;381(21):2020-31.

5. Mok TSK, Wu YL, Kudaba I, et al. Pembrolizumab versus chemotherapy for previously untreated, PD-L1-expressing, locally advanced or metastatic non-small-cell lung cancer (KEYNOTE-042): a randomised, open-label, controlled, phase 3 trial. Lancet. 2019;393(10183):1819-30.

6. Reck M, Rodriguez-Abreu D, Robinson AG, et al. Pembrolizumab versus chemotherapy for PD-L1-positive non-small-cell lung cancer. N Engl J Med. 2016;375(19):1823-33.

7. Signorovitch JE, Wu EQ, Yu AP, et al. Comparative effectiveness without head-to-head trials: a method for matching-adjusted 
indirect comparisons applied to psoriasis treatment with adalimumab or etanercept. Pharmacoeconomics. 2010;28(10):935-45.

8. Bucher HC, Guyatt GH, Griffith LE, Walter SD. The results of direct and indirect treatment comparisons in meta-analysis of randomized controlled trials. J Clin Epidemiol. 1997;50(6):683-91.

9. Guyot P, Ades AE, Ouwens MJNM, Welton NJ. Enhanced secondary analysis of survival data: reconstructing the data from published Kaplan-Meier survival curves. BMC Med Res Methodol. 2012;12(1):9.

10. Halmos B, Burke T, Kalyvas C, et al. An indirect comparison of pembrolizumab monotherapy vs Ipilimumab + Nivolumab for first line metastatic NSCLC with PD-L1 TPS $\geq 1 \%$. In: The International Association for the study of lung cancer (IASLC) 2020 North America conference on lung cancer; 16-17 October 2020: Chicago, IL

11. Zhou Y, Zhang Y, Guo G, et al. Nivolumab plus ipilimumab versus pembrolizumab as chemotherapy-free, first-line treatment for PD-L1-positive non-small cell lung cancer. Clin Transl Med. 2020;10(1):107-15.

12. Qiao N, Insinga R, de Junior GLL, Cook J, Sénécal M. A review of cost-effectiveness studies of Pembrolizumab regimens for the treatment of advanced non-small cell lung cancer. Pharmacoeconomics Open. 2021. https://doi.org/10.1007/s41669-020-00255-2 (Epub 19 Jan 2021).

13. Huang M, Lou Y, Pellissier J, et al. Cost effectiveness of pembrolizumab vs. standard-of-care chemotherapy as first-line treatment for metastatic NSCLC that expresses high levels of PD-L1 in the United States. Pharmacoeconomics. 2017;35(8):831-44.

14. Huang M, Lopes GL, Insinga RP, et al. Cost-effectiveness of pembrolizumab versus chemotherapy as first-line treatment in PD-L1-positive advanced non-small-cell lung cancer in the USA. Immunotherapy. 2019;11(17):1463-78.

15. She $\mathrm{L}, \mathrm{Hu} \mathrm{H}$, Liao $\mathrm{M}$, et al. Cost-effectiveness analysis of pembrolizumab versus chemotherapy as first-line treatment in locally advanced or metastatic non-small cell lung cancer with PD-L1 tumor proportion score $1 \%$ or greater. Lung Cancer. 2019;138:88-94.

16. Weng X, Luo S, Lin S, et al. Cost-utility analysis of pembrolizumab versus chemotherapy as first-line treatment for metastatic non-small cell lung cancer with different PD-L1 expression levels. Oncol Res. 2020;28(2):117-25.

17. Berling M, Chaudhary MA, Yuan Y, et al. Cost effectiveness analysis of nivolumab plus ipilimumab in the first line treatment of metastatic non small cell lung cancer in the United States. In: The International Association for the Study of Lung Cancer (IASLC) 2020 North American conference on lung cancer, 16-17 Oct 2020; Chicago, IL

18. Courtney PT, Yip AT, Cherry DR, Salans MA, Kumar A, Murphy JD. Cost effectiveness of combination ipilimumab-nivolumab in advanced non-small-cell lung cancer. J Clin Oncol. 2020;38(15 Suppl):e19387-e19387.

19. Hu H, She L, Liao M, et al. Cost-effectiveness analysis of nivolumab plus ipilimumab vs. chemotherapy as first-line therapy in advanced non-small cell lung cancer. Front Oncol. 2020;10:1649-1649.
20. Li J, Zhang T, Xu Y, et al. Cost-effectiveness analysis of nivolumab plus ipilimumab versus chemotherapy as first-line treatment in advanced NSCLC. Immunotherapy. 2020;12(14):1067-75.

21. US FDA. YERVOY (ipilimumab) injection, for intravenous use. 2020. https://www.accessdata.fda.gov/drugsatfda_docs/label/ 2020/125377s119lbl.pdf. Accessed 15 Mar 2021

22. Wong W, Carlson J, Cloutier M. Estimating the Costs of Adverse Events in Economic Models: Is There a "Right" Approach? Value \& Outcomes Spotlight. 2019:27-29. https://www.ispor.org/docs/ default-source/publications/value-outcomes-spotlight/mayjune-2019/heor-article---william-wong.pdf?sfvrsn=f0c0af42_0. Accessed 9 Mar 2021.

23. Medicare.gov. Medicare costs at a glance. 2021. https://www. medicare.gov/your-medicare-costs/medicare-costs-at-a-glance. Accessed 9 Jul 2021

24. Sanders GD, Neumann PJ, Basu A, et al. Recommendations for conduct, methodological practices, and reporting of cost-effectiveness analyses: second panel on cost-effectiveness in health and medicine. JAMA. 2016;316(10):1093-103.

25. US Bureau of Labor Statistics. Table 1: Consumer Price Index for All Urban Consumers (CPI-U): US city average, by expenditure category. 2020. https://www.bls.gov/news.release/cpi.t01.htm. Accessed 16 Mar 2020

26. First DataBank. FDB MedKnowledge Drug Database. https:// www.fdbhealth.com/solutions/medknowledge-drug-database. Accessed 2 Jul 2020

27. Centers for Medicare and Medicaid Services. FY2020 IPPS Final Rule Home Page. 2020. https://www.cms.gov/Medicare/Medic are-Fee-for-Service-Payment/AcuteInpatientPPS/FY2020-IPPSFinal-Rule-Home-Page. Accessed 4 Mar 2021

28. Lopez E, Neuman T, Jacobson G, Levitt L. How much more than medicare do private insurers pay? A review of the literature. https://www.kff.org/medicare/issue-brief/how-much-more-thanmedicare-do-private-insurers-pay-a-review-of-the-literature/. Accessed 15 Apr 2020

29. The Kaiser Family Foundation. Medicaid-to-Medicare Fee Index. 2016. https://www.kff.org/medicaid/state-indicator/medicaid-tomedicare-fee-index/?currentTimeframe $=0 \&$ sortModel $=\% 7 \mathrm{~B} \%$ 22colId \%22:\%22Location \%22,\%22sort $\% 22: \% 22$ asc $\% 22 \% 7 \mathrm{D}$. Accessed 9 Jul 2021

30. Hariton E, Locascio JJ. Randomised controlled trials-the gold standard for effectiveness research: Study design: randomised controlled trials. BJOG. 2018;125(13):1716-1716.

31. Phillippo DM, Ades AE, Dias S, Palmer S, Abrams KR, Welton NJ. Methods for population-adjusted indirect comparisons in health technology appraisal. Med Decis Mak. 2018;38(2):200-11.

32. Signorovitch JE, Sikirica V, Erder MH, et al. Matching-adjusted indirect comparisons: a new tool for timely comparative effectiveness research. Value Health. 2012;15(6):940-7.

33. Boyer M, Şendur MAN, Rodríguez-Abreu D, et al. Pembrolizumab plus ipilimumab or placebo for metastatic non-small-cell lung cancer with PD-L1 tumor proportion score $\geq 50 \%$ : randomized, double-blind phase III KEYNOTE-598 study. J Clin Oncol. 2021;39(21):2327-38 (JCO.20.03579). 